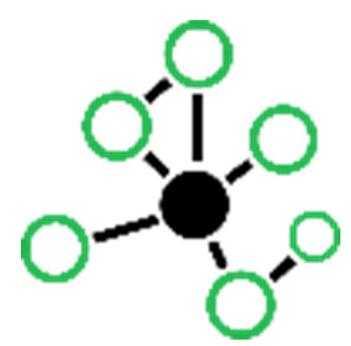

\title{
Last Frontier of Agricultural Big Data in Rotation?
}

\author{
Keizer, P. ${ }^{1}$, S. Schnable ${ }^{1}$, M. Riemens ${ }^{1}$ \\ ${ }^{1}$ Wageningen University \& Research, Droevendaalsesteeg 1, 6708 PB Wageningen, the Netherlands \\ Corresponding author's e-mail: paul.keizer@wur.nl
}

Over the years new and promising varieties are bred not only for resistance to diseases but also for direct yield by stress tolerance, architectural or other properties. However, these potential yield improvements under optimal circumstances are not met by the growers in their practice, and is even widening.

To investigate the contribution of management strategies to the yield gap, a Rotation dataset was built on observed data collected over 10 years. This realistic and ever growing data set was prospected for combination of elements that are most relevant for the yield gap. Since the data contain mostly hand-filled fields careful curation is essential. In addition, observational data are less information-dense than results of designed experiments, therefore one needs large amounts of data before one can spot trends.

The target is to sort-out variables, sensitive to management in what en when, that bear importance, corrected for the effect of other more static variables.

The final step in the analysis, Stochastic Frontier Analysis, combines the variables in an optimal way to guide growers in taking management decisions given other external factors like the weather.

In this study it is tried to address the shortcomings, methods used and potential of such Big Data in the environment of growers, whom in the near future will be dealing with an overwhelming amount of information and have to decide where to focus on, giving their natural restrictions of sustainable intensification of food production without too much an investment. 